The Astrophysical Journal, 683:238-247, 2008 August 10

(C) 2008. The American Astronomical Society. All rights reserved. Printed in U.S.A.

\title{
THE DIFFERENT STRUCTURES OF THE TWO CLASSES OF STARLESS CORES
}

\author{
ERIC KeTO \\ Harvard-Smithsonian Center for Astrophysics, 60 Garden Street, Cambridge, MA 02138; keto@cfa.harvard.edu \\ AND \\ Paola Caselli \\ School of Physics and Astronomy, University of Leeds, Leeds LS2 9JT, UK; p.caselli@1eeds.ac.uk \\ Received 2008 February 25; accepted 2008 April 7
}

\begin{abstract}
We describe a model for the thermal and dynamical equilibrium of starless cores that includes the radiative transfer of gas and dust and simple CO chemistry. The model shows that the structure and behavior of the cores is significantly different depending on whether the central density is either above or below about $10^{5} \mathrm{~cm}^{-3}$. This density is significant as the critical density for gas cooling by gas-dust collisions and as the critical density for dynamical stability, given the typical properties of the starless cores. Starless cores thus divide into two classes that we refer to as thermally supercritical and thermally subcritical. This two-class distinction allows an improved interpretation of the different observational data of starless cores within a single model.
\end{abstract}

Subject heading: ISM: clouds

\section{INTRODUCTION}

Starless cores are dense regions $\left(n_{\mathrm{H}_{2}} \sim 10^{4}-10^{6} \mathrm{~cm}^{-3}\right)$ in dark clouds with linear scales of tenths of parsecs, and total masses of a few solar masses. The starless cores contain no infrared sources above the sensitivity level of the IRAS satellite (about $0.1 L_{\odot}$ at the distance of Taurus) and thus are thought to be sites of possible future rather than current star formation (Myers et al. 1983; Myers \& Benson 1983; Benson \& Myers 1989; Beichman et al. 1986; Ward-Thompson et al. 1994, 1999; Tafalla et al. 1998; Lee \& Myers 1999; Bacmann et al. 2000; Shirley et al. 2000; Lee et al. 2001; di Francesco et al. 2007; Bergin \& Tafalla 2007). Detailed observations of individual starless cores (Alves et al. 2001; Tafalla et al. 2004) show density profiles that approximately match those of pressure-confined, hydrostatic spheres (Bonnor-Ebert or BE spheres; Bonnor 1956).

Despite an overall similarity in structure, recent observations with increasingly better angular resolution and sensitivity show that starless cores are not all the same. For example, observations of L1517B indicate isothermal gas and show spectral line profiles indicative of little motion or possibly expansion (Tafalla et al. 2004; Keto et al. 2004; Sohn et al. 2007). In contrast, observations of L1544 indicate a significant variation in temperature from center to edge (Crapsi et al. 2007) and show spectral line profiles consistent with an overall contraction (Williams et al. 1999; Caselli et al. 2002; Keto et al. 2004; Sohn et al. 2007). The cores B68 and TMC-1C provide another example in contrast. Observations of $\mathrm{CO}$ in $\mathrm{B} 68$ indicate an excitation temperature rising toward the edge of the core (Bergin et al. 2006) and show spectral line profiles consistent with internal oscillations (sound waves; Lada et al. 2003). In comparison, observations of CO isotopologues in TMC-1C (Schnee et al. 2007) indicate an excitation temperature decreasing toward the edge and show spectral line profiles consistent with contraction.

In an earlier paper (Keto \& Field 2005) we suggested that the observed differences were consistent with the different temperature and density structures of starless cores that have central densities greater or less than about $10^{5} \mathrm{~cm}^{-3}$. The significance of this particular density is twofold.
First, this density divides the starless cores into those that are dynamically stable (those with lower densities) and those that must be contracting or collapsing (those with higher densities). The dynamical stability, of course, also depends on the total mass and the internal energy of the core, but for starless cores with masses of $1-10 M_{\odot}$, temperatures of around $10 \mathrm{~K}$, and subsonic internal velocities, the critical density for dynamical stability falls within a range around $10^{5} \mathrm{~cm}^{-3}$.

Second, at densities below a few $10^{5} \mathrm{~cm}^{-3}$ the molecular gas in starless cores cools primarily through molecular line radiation, but at higher densities the gas cools primarily through collisional coupling with dust. This difference in cooling results in different temperature structures in cores with central densities below or above $10^{5} \mathrm{~cm}^{-3}$. If the density in a core is everywhere below $10^{5} \mathrm{~cm}^{-3}$, so that the gas and dust are nowhere collisionally coupled, then the entire core cools primarily by molecular line radiation. As a result, the core is approximately isothermal. If the core has a central density above $10^{5} \mathrm{~cm}^{-3}$, the gas in the core center cools by collisional coupling with the dust, whereas the gas elsewhere cools by molecular line radiation. This difference in cooling results in a variation in temperature across the core. Because the dust, heated by external starlight, is cooler in the core center, the gas there, coupled to the dust, is also cooler.

In this paper we continue our investigation of the structures of the two classes of starless cores. We improve our previous model of thermal equilibrium by accounting for the heating of the gas by hot electrons photoelectrically released from dust grains and by including variable molecular abundances based on a simple model for $\mathrm{CO}$ chemistry. Our improved model for starless cores allows a better interpretation of the observational data.

For example, the inclusion of photoelectric heating provides a better description of the thermal structure near the core boundary where this effect is most significant. Applied to recent observations of the edges of the starless cores B68 and TMC-1C (Bergin et al. 2006; Schnee et al. 2007), the rising and falling $\mathrm{CO}$ excitation temperature seen in the observations of the two cores, respectively, are consistent with the different effect of photoelectric heating on cores with relatively higher and lower densities at their boundaries. 
The loss from the gas phase of $\mathrm{CO}$ and other molecular coolants has only a minor effect on the gas temperature and hence the density structure of starless cores (Goldsmith 2001). Nonetheless, an understanding of the molecular abundances is quite important in the interpretation of spectral line observations, particularly the line profiles and strengths. We have used our simple model of $\mathrm{CO}$ chemistry in modeling spectral line profiles in an oscillating lower mass, lower density starless core (Broderick et al. 2007) and in modeling spectral line strengths in a collapsing, higher mass, higher density, starless core (E. Keto \& P. Caselli, in preparation).

Our improved models of starless cores are also useful in constraining some of the properties of the interstellar medium (ISM) that relate to the thermal equilibrium of the starless cores. In particular, we obtain temperatures closer to those indicated by observations with dust opacities higher than those of naked grains and more consistent with fluffy dust (Ossenkopf \& Henning 1994; Kruegel \& Siebenmorgen 1994) and cosmic-ray ionization rate toward the lower end of the range of estimates, $1 \times 10^{-17} \mathrm{~s}^{-1}$ to $6 \times 10^{-17} \mathrm{~s}^{-1}$. We find that photoelectric heating improves the stability of cores by increasing the confining pressure with reduced overlying mass, but additional internal energy is still required if the larger cores are to be supported.

\section{THE TWO CLASSES OF STARLESS CORES}

\subsection{Observational Description}

The higher mass and higher density cores are unstable to collapse and the formation of protostars within a free-fall time but, being starless, these cores have not yet formed a protostar. Thus these cores may be thought of as "young brides" that are soon to give birth to stars. The density profiles within these cores are more centrally concentrated, owing to the greater importance of gravitational forces in confining the core. These cores often show asymmetric spectral line profiles (Williams et al. 1999; Gregerson et al. 1997; Launhardt et al. 1998; Lee et al. 1999; Gregersen \& Evans 2000; Caselli et al. 2002; Keto et al. 2004; Lee et al. 2004a, 2004b; Sohn et al. 2004), consistent with inward motions, and line widths that increase toward the centers of the cores, consistent with inward acceleration of the infall. Spectral lines from some cores show two components separated by more than the sound speed, indicating supersonic velocities (Sohn et al. 2007). These denser cores are not isothermal. Observations of both dust (WardThompson et al. 2002; Schnee \& Goodman 2005; Pagani et al. 2003, 2004) and gas (Crapsi et al. 2007; Pagani et al. 2007) indicate cooler temperatures in their centers, the latter consistent with the change from molecular line cooling to more efficient dust cooling as the density increases toward the interior.

In contrast, lower mass and lower density cores are stable against gravitational contraction, and therefore in the absence of changes in their environment, will remain starless. These barren cores may be thought of as "old maids." These cores have flatter density profiles consistent with the relatively greater importance of an external pressure in confining the cores. Their spectral line profiles may be simple Gaussians indicating little internal motion or show complex shapes consistent with internal oscillations (sound waves; Lada et al. 2003; Redman et al. 2006; Aguti et al. 2007; Maret et al. 2007). Multiwavelength infrared observations also indicate internal density perturbations (Steinacker et al. 2005a, $2005 b)$. The line widths are generally more constant across the core and indicate subsonic internal velocities. These cores are more nearly isothermal $(\sim 10 \mathrm{~K})$, consistent with molecular line cooling throughout.

Some recent papers have also drawn attention to differences within the population of starless cores and use terms such as "prestellar" to indicate a tendency toward collapse and star formation versus "starless" or "failed" to suggest the opposite (André et al. 2008). Sometimes the distinction is drawn as "gravitationally bound" and "gravitationally unbound," suggesting a ratio of greater or less than unity of gravitational energy to internal energy, including thermal energy and possibly microturbulence and magnetic energy. These terms suggest a division of the starless cores into two classes distinguished by their gravitational stability and future evolution. Kirk et al. (2005) divide the starless cores into two categories called "bright" and "intermediate." These refer to the brightness of the dust emission which is related to the mass and density of the cores. In this paper we suggest that the distinction is motivated by other observational properties as well, and that the various properties of the two classes may be derived from a single theoretical model for the starless cores that we describe in the following sections.

\subsection{Theoretical Description: Thermally Supercritical and Thermally Subcritical}

We refer to the two classes of cores as "thermally supercritical" and "thermally subcritical." We choose these terms to emphasize that the distinction is based on the physics of the cores rather than their origin or evolution. The term "thermal" in this description is appropriate because it refers to both their thermalradiative equilibrium and to their thermally supported dynamical equilibrium. Whether the cores are supported primarily by thermal energy or by magnetic energy is still a matter of debate. The evidence is gathering on the side of thermal support with nonthermal energy being an important but minor contributor to their stability (Barranco \& Goodman 1998; Goodman et al. 1998; Tafalla et al. 2004; Lada et al. 2008). In other words, without any nonthermal energy most of the cores would be unstable. However, to achieve stability, the cores require only an amount of nonthermal energy that is a fraction of their thermal energy. The terms thermally super- and subcritical are similar in meaning to the terms magnetically super- and subcritical that are well established in the literature, but the "thermal" modifier suggests that thermal energy is here more important than magnetic energy. It is not necessary to prove this supposition to adopt the terminology, and in any case, in this study we model the cores as thermally supported.

As typical parameters for our calculations, we adopt a central density of $10^{6} \mathrm{~cm}^{-3}$ and a total mass of $10 M_{\odot}$ to represent a thermally supercritical core, and $10^{5} \mathrm{~cm}^{-3}$ and $1 M_{\odot}$ for a thermally subcritical core. The well-studied cores L1544 and B68 are estimated to have similar central densities. Crapsi et al. (2007) estimate $2 \times 10^{6} \mathrm{~cm}^{-3}$ for L1544 and Keto et al. (2006) and Bergin et al. (2006) estimate $2.5 \times 10^{5} \mathrm{~cm}^{-3}$ and $3.0 \times 10^{5} \mathrm{~cm}^{-3}$, respectively, for B68; all three estimates are based on fitting isothermal BE spheres to the observational data. While L1544 and B68 are prototypes of cores in the two classes, here we are not attempting to model these two cores exactly. Our example parameters are chosen as round numbers that provide a factor of 10 difference in density and mass, sufficient to bring out the differences in their structures. Of course, the real cores have combinations of mass and density that form a continuum that includes these two examples. Other parameters for the cores are listed in Table 1.

\section{CO CHEMISTRY}

Molecular abundances in starless cores are the result of complex interactions involving gas-phase chemistry, freezeout and desorption, and photodissociation. In order to develop a simplified model we assume that the abundance of $\mathrm{CO}$ is decreased by two 
TABLE 1

Parameters of Models Illustrated in the Figures

\begin{tabular}{|c|c|c|c|c|c|}
\hline Figure & Core & Cosmic-Ray Ionization Rate & Photoelectric Heating & Depletion & Dust Opacity \\
\hline 3.….................... & YB & Low & Off & Yes & $1 \times$ \\
\hline 4 & $\mathrm{OM}$ & Isothermal & Isothermal & Yes & $1 \times$ \\
\hline 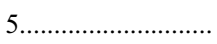 & YB & Low & Off & Both & $1 \times$ \\
\hline 6........................... & $\mathrm{OM}$ & Low & Off & Both & $1 \times$ \\
\hline 7 & YB & Low & On & Yes & $1 \times$ \\
\hline 8 & OM & Low & On & Yes & $1 \times$ \\
\hline 9......................... & YB & Low & On & Yes & $1 \times$ \\
\hline $10 \ldots \ldots \ldots \ldots \ldots \ldots \ldots$ & OM & Low & On & Yes & $1 \times$ \\
\hline $11 \ldots \ldots \ldots \ldots \ldots \ldots \ldots$ & OM, YB & Low & On & Yes & $1 \times$ \\
\hline \multirow[t]{4}{*}{$12 \ldots \ldots \ldots \ldots \ldots \ldots \ldots \ldots$} & YB & Low & On & Yes & $1 \times$ \\
\hline & $\ldots$ & Std & On & Yes & $4 \times$ \\
\hline & $\ldots$ & Std & On & Yes & $1 \times$ \\
\hline & $\ldots$ & High & On & Yes & $1 \times$ \\
\hline $13 \ldots \ldots \ldots \ldots \ldots \ldots \ldots$ & $\mathrm{OM}$ & Same as Fig. 12 & $\ldots$ & $\ldots$ & $\ldots$ \\
\hline $14 \ldots \ldots \ldots \ldots \ldots \ldots \ldots \ldots$ & YB & Low & On, off & Yes & $1 \times$ \\
\hline
\end{tabular}

Notes.-YB = supercritical; $\mathrm{OM}=$ subcritical; low $=1.3 \times 10^{-17} \mathrm{~s}^{-1} ; \mathrm{std}=3.0 \times 10^{-17} \mathrm{~s}^{-1} ;$ high $=6.0 \times 10^{-17} \mathrm{~s}^{-1}$; isothermal $=$ core structure from Broderick et al. (2007); $1 \times=$ dust opacities of Ossenkopf \& Henning (1994); $4 \times=$ dust opacities 4 times higher; both $=$ figure compares structures with depleted and undepleted abundances.

effects: (1) freezeout onto dust grains in the high density center, and (2) photodissociation at the edge of the core.

\subsection{The Photodissociation Region}

Tielens \& Hollenbach (1985) suggest that at the edge of a molecular cloud where photodissociation is important, the dominant cycle for the formation and destruction of $\mathrm{CO}$ is

$$
\mathrm{C}^{+} \stackrel{\mathrm{H}_{2}}{\rightarrow} \mathrm{CH}_{2} \underset{\mathrm{O}}{\rightarrow} \mathrm{CO} \stackrel{h \nu}{\rightarrow} \mathrm{C} \stackrel{h \nu}{\rightarrow} \mathrm{C}^{+}
$$

The timescale for the formation of $\mathrm{CH}_{2}$ by radiative association is longer than for the formation of $\mathrm{CO}$ from $\mathrm{CH}_{2}$ and $\mathrm{O}$. Therefore we assume that the rate of formation of $\mathrm{CO}$ is given by that of $\mathrm{CH}_{2}$. This eliminates consideration of the complex chemistry of oxygen. The $\mathrm{CO}$ cycle may then be described by three rate equations for the creation and destruction of $\mathrm{C}^{+}, \mathrm{C}$, and $\mathrm{CO}$, for example

$$
\frac{d \mathrm{C}^{+}}{d t}=-R_{D}\left(\mathrm{C}^{+}\right)+R_{C}\left(\mathrm{C}^{+}\right)
$$

where $R_{D}$ and $R_{C}$ are the rates for the destruction and creation of $\mathrm{C}^{+}$. Similar equations hold for $\mathrm{C}$ and $\mathrm{CO}$. The conservation equation in the form

$$
\mathrm{C}^{+}+\mathrm{CO}+\mathrm{C}=1
$$

indicates that the symbols stand for the nondimensional relative abundance of the three species with respect to the total abundance of carbon. Assuming steady state we may solve for the relative abundance of $\mathrm{CO}$ as

$$
\mathrm{CO}=\left(\frac{\mathrm{CO}}{\mathrm{C}^{+}}\right)\left(1+\frac{\mathrm{CO}}{\mathrm{C}^{+}}+\frac{\mathrm{C}}{\mathrm{C}^{+}}\right)^{-1}
$$

The ratios of the relative abundances, $\mathrm{CO} / \mathrm{C}^{+}$and $\mathrm{C} / \mathrm{C}^{+}$, are equal to the ratios of the rates of destruction and creation of the respective species in the numerators. Using the data in Tielens \& Hollenbach (1985), these ratios are

$$
\frac{\mathrm{CO}}{\mathrm{C}^{+}}=\frac{1.4 \times 10^{-11} G_{0} \exp \left(-3.2 A_{V}\right)}{2.1 \times 10^{-10} G_{0} \exp \left(-2.6 A_{V}\right)}
$$

and

$$
\frac{\mathrm{C}}{\mathrm{C}^{+}}=\frac{n\left(\mathrm{H}_{2}\right) 6 \times 10^{-16}}{2.1 \times 10^{-10} G_{0} \exp \left(-2.6 A_{V}\right)} .
$$

Here, $n\left(\mathrm{H}_{2}\right)$ is the number density of hydrogen, $G_{0}$ is the interstellar radiation field in units of the Habing flux (Habing 1968), $A_{V}$ is the mean visual extinction to the interstellar radiation field, and the numerical values of the coefficients are taken from Tables 5 and 12 of Tielens \& Hollenbach (1985). The mean extinction at each point in the cloud is computed as the extinction from the point to the cloud surface averaged over all directions,

$$
\left\langle\exp \left(-A_{V}\right)\right\rangle=\frac{1}{4 \pi} \int \exp \left(-A_{V}\right) d \Omega .
$$

The Habing flux $G_{0}=1$ corresponds to the average interstellar radiation field of the Galaxy. Figure 1 shows the variation of the three species, $\mathrm{CO}, \mathrm{C}$, and $\mathrm{C}^{+}$as a function of $A_{V}$ for a cloud with a constant density of $1000 \mathrm{~cm}^{-3}$ and $G_{0}=1$. For this flux level, the simple model provides for exponential replacement of $\mathrm{CO}$ and $\mathrm{C}^{+}$at an $A_{V}$ of about 1 . The more complex photodissociation model of Tielens \& Hollenbach (1985) shows that this exponential replacement is the dominant relationship, although in their Figure $9 b$ the replacement occurs at a higher $A_{V}$ of $3-4$ because of the higher radiative flux in their model, $G_{0}=10^{5}$.

\subsection{Freezeout}

By comparing dust and molecular line emission, Willacy et al. (1998), Bergin et al. (2001), Caselli et al. (1999, 2002), Bacmann et al. (2002, 2003), Hotzel et al. (2002), Bergin et al. (2002), Tafalla et al. (1998, 2002, 2004, 2006), Redman et al. (2002, Crapsi et al. (2004, 2005), Pagani et al. (2005), Schnee et al. (2007), and Carolan et al. (2008) were able to measure variations in the gas-phase abundance of several molecules in several cold, 


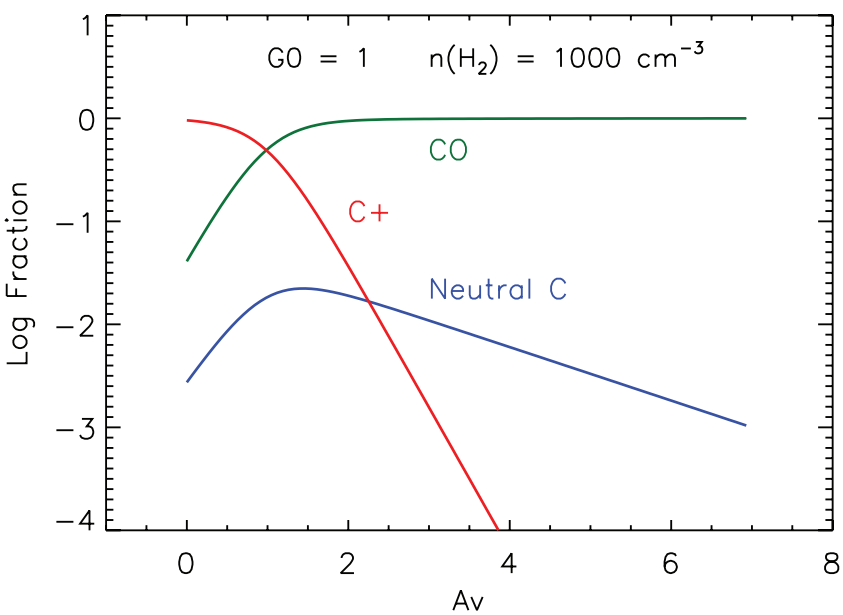

FIG. 1.-Variation in the abundances of $\mathrm{C}, \mathrm{CO}$, and $\mathrm{C}^{+}$in the photodissociation region at the edge of a cloud as calculated by the simple model described in the text. In this figure, the cloud has a constant density of $1000 \mathrm{~cm}^{-3}$ and the interstellar radiation field has a Habing flux, $G_{0}=1$.

dark clouds. The observations suggest that the molecular abundance in dense gas is an equilibrium between the rate of depletion from the gas phase as molecules freeze onto dust grains and the rate of the inverse process of desorption (Brown et al. 1988; Hasegawa et al. 1992; Bergin et al. 1995; Aikawa et al. 2001, 2003, 2005; Li et al. 2002; Pavlyuchenkov et al. 2003; Shematovich et al. 2003; Lee et al. 2004). This suggests that the steady state abundance of $\mathrm{CO}$ in dense gas may be computed as the ratio of the depletion time to the sum of the depletion and desorption times.

The timescale for depletion onto dust may be estimated (Rawlins et al. 1992)

$$
\tau_{\mathrm{on}}=\left[S_{0} R_{\mathrm{dg}} n\left(\mathrm{H}_{2}\right) \sigma V_{T}\right]^{-1}
$$

Here $S_{0}$ is the sticking coefficient, with $S_{0}=1$ meaning that the molecule sticks to the dust in each collision; $R_{\mathrm{dg}}$ is the ratio of the number density of dust grains relative to molecular hydrogen; $\sigma$ is the mean cross section of the dust grains; and $V_{T}$ is the relative velocity between the grains and the gas. If the grains have a power-law distribution of sizes with the number of grains of each size scaling as the -3.5 power of their radii (Mathis et al. 1977), then we can estimate their mean cross section as

$$
\langle\sigma\rangle=\left[\int_{a_{1}}^{a_{2}} n(a) d a\right]^{-1} \int_{a_{1}}^{a_{2}} n(a) \sigma(a) d a
$$

where $a_{1}$ and $a_{2}$ are the minimum and maximum grain sizes. If $a_{1}=0.005 \mu \mathrm{m}$ and $a_{2}=0.3 \mu \mathrm{m}$, then $\langle\sigma\rangle=3.4 \times 10^{-4} \mu \mathrm{m}^{2}$. Similarly, the ratio of the number densities of dust and gas may be estimated by computing the mean mass of a dust grain and assuming the standard gas to dust mass ratio of 100 . If the density of the dust is $2 \mathrm{~g} \mathrm{~cm}^{-3}$, then the ratio of number densities is $R_{\mathrm{dg}}=4 \times 10^{-10}$. The relative velocity due to thermal motion is

$$
V_{T}=\left(\frac{8 k T}{\pi \mu}\right)^{-1 / 2}
$$

where $T$ is the temperature and $\mu$ the molecular weight.

There are several processes that generate heat on dust grains that can liberate frozen molecules to the gas phase. These include exothermic $\mathrm{H}_{2}$ formation on the grains, cosmic-ray collisions with

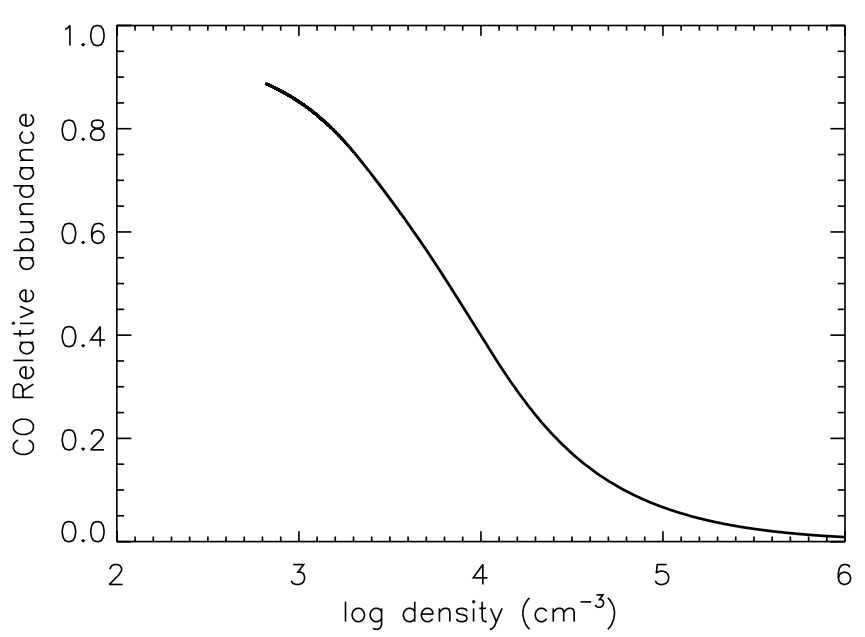

FIG. 2.- Relative abundance of $\mathrm{CO}$ in the gas phase in equilibrium between depletion and desorption as a function of density. The depletion is caused by the loss of CO from the gas phase as the molecules freeze onto dust grains (eq. [12] in text). The depletion is a function of density because the freezeout is proportional to the dust-gas collision rate and inversely proportional to the cosmic-ray-induced desorption rate, which is itself independent of density.

dust grains, and photodesorption of molecules off the grains (Willacy \& Williams 1993). The UV radiation for this latter process derives from the ionization of the molecular gas by cosmic rays. According to Roberts et al. (2007), in starless cores these processes are all independent of density. The rate of $\mathrm{H}_{2}$ formation is independent of the molecular density because the density of $\mathrm{H}$ i in starless cores is independent of the molecular density. The rates of all these desorption processes are not well defined, nor is it known which is dominant. Therefore, for definiteness we adopt the rate given by Hasegawa \& Herbst (1993) for desorption by cosmic rays but with the understanding that other processes might also be important. The timescale is

$$
\tau_{\text {off }}=3.3 \times 10^{6}\left(\frac{\zeta}{10^{-17}}\right)^{-1} \exp \left(E_{\mathrm{CO}} / 70\right)(\mathrm{yr})
$$

where the cosmic-ray ionization rate $\zeta=3 \times 10^{-17} \mathrm{~s}^{-1}$, and the binding energy of $\mathrm{CO}$ onto ice is $E_{\mathrm{CO}}=1100 \mathrm{~K}$ (Öberg et al. 2005, 2007). We choose this value for the binding energy because it is between the binding energies of CO on water ice, $1180 \mathrm{~K}$ (Collins et al. 2003a, 2003b), and on CO ice, $850 \mathrm{~K}$ (Fraser et al. 2001), assuming a typical solid $\mathrm{H}_{2} \mathrm{O} / \mathrm{CO}$ abundance ratio of $\simeq 30$ on ice mantles in dense clouds (Whittet et al. 2007).

The relative abundance of $\mathrm{CO}$ in the gas phase in equilibrium between depletion and desorption is

$$
\mathrm{CO}_{\text {gas }}=\frac{\tau_{\text {on }}}{\tau_{\text {on }}+\tau_{\text {off }}},
$$

where $\mathrm{CO}_{\text {gas }}+\mathrm{CO}_{\text {grains }}=1$, and the symbols stand for the nondimensional relative abundance of $\mathrm{CO}$ in the two phases with respect to the total $\mathrm{CO}$ abundance. The depletion time scales inversely with the collision rate while the desorption time scales inversely with the cosmic-ray ionization rate. Therefore the depletion of $\mathrm{CO}$ is dependent only on the gas density because the cosmic-ray ionization rate is assumed to be the same throughout the cloud. Figure 2 shows the dependence of the steady state abundance of $\mathrm{CO}$ versus the molecular gas density.

While we only illustrate the steady state abundance of $\mathrm{CO}$ in this paper, the model for depletion and desorption may also be used to describe the time-dependent evolution of $\mathrm{CO}$ between 


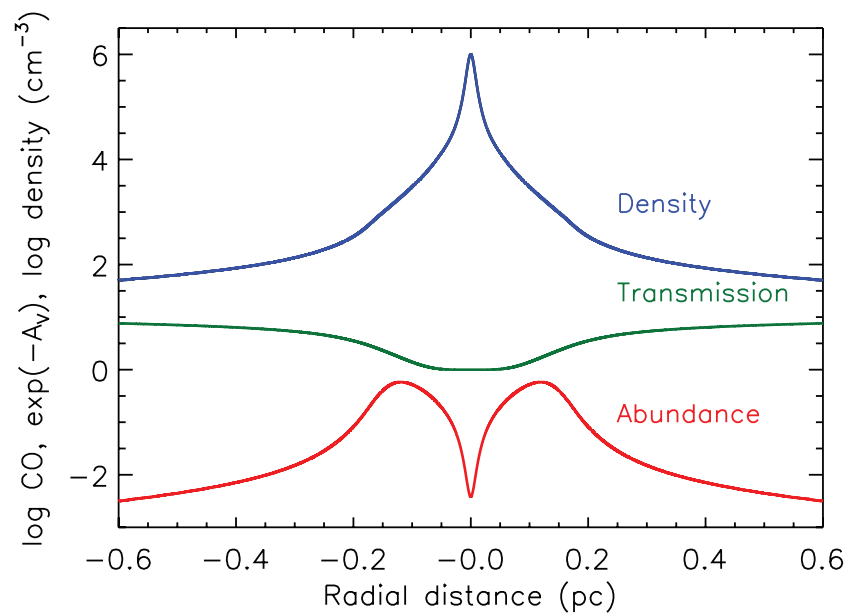

FIG. 3.-Structure of a thermally supercritical core. The figure shows the CO abundance, mean extinction, and gas density of a model cloud with the parameters of a thermally supercritical core. The model cloud is a BE sphere similar to that in Fig. 5 of Keto \& Field (2005). The lowest curve is the log of the relative CO abundance. A value of zero represents no reduction in $\mathrm{CO}$ abundance from photodissociation or depletion. The middle curve is the transmission of starlight. A value of 1 represents no extinction and zero represents total extinction. The top curve is the $\log$ of the number density. Although the maximum radius on the plot is $0.6 \mathrm{pc}$, the core would not be observed to have this extent because of the low density and molecular abundance at large radii. Parameters are listed in Table 1.

the gas and grain phases. The change with time of the abundance in the gas phase is

$$
\frac{d \mathrm{CO}_{\text {gas }}}{d t}=-\frac{\mathrm{CO}_{\text {gas }}}{\tau_{\text {on }}}+\frac{\mathrm{CO}_{\text {grain }}}{\tau_{\text {off }}} .
$$

\subsection{CO Abundances in Model Cores}

The combined effects of photodissociation and depletion result in the abundance of $\mathrm{CO}$ peaking inside the boundary of the core where the visual extinction is just high enough to shield the $\mathrm{CO}$, but the density has not yet become high enough for significant depletion by freezeout. This effect is seen in Figures 3 and 4. Figure 3 shows a spherically symmetric, static-equilibrium model with the properties of a thermally supercritical core. This core is the same as shown in Figure 5 of Keto \& Field (2005) but with a factor of 2 higher total mass. Figure 4 shows a stable, starless core perturbed by an internal oscillation. This particular structure of an oscillating core, motivated by observations of B68, is the same as illustrated in Figures 6 and 7 of Broderick et al. (2007) and is included here to show how the abundance varies in response to an asymmetric density profile. The sharp drop in density at the boundary of this thermally subcritical core is due to the high internal density at the boundary where the core is truncated to give it a low mass. In B68, this transition implies a high-pressure exterior confining gas, probably hot gas.

\section{ENERGY BALANCE AND TEMPERATURE}

Models for the temperature structure of dark clouds have been described in a number of papers (Larson 1973, 1985; Clarke \& Pringle 1997; Evans et al. 2001; Shirley et al. 2002; Zucconi et al. 2001; Stamatellos \& Whitworth 2003; Goncalves et al. 2004; Keto \& Field 2005). These models include the effects of molecular line cooling, the radiative equilibrium of dust in starlight, dust-gas collisional coupling, and cosmic-ray heating. We start with the model described in Keto \& Field (2005) that includes these

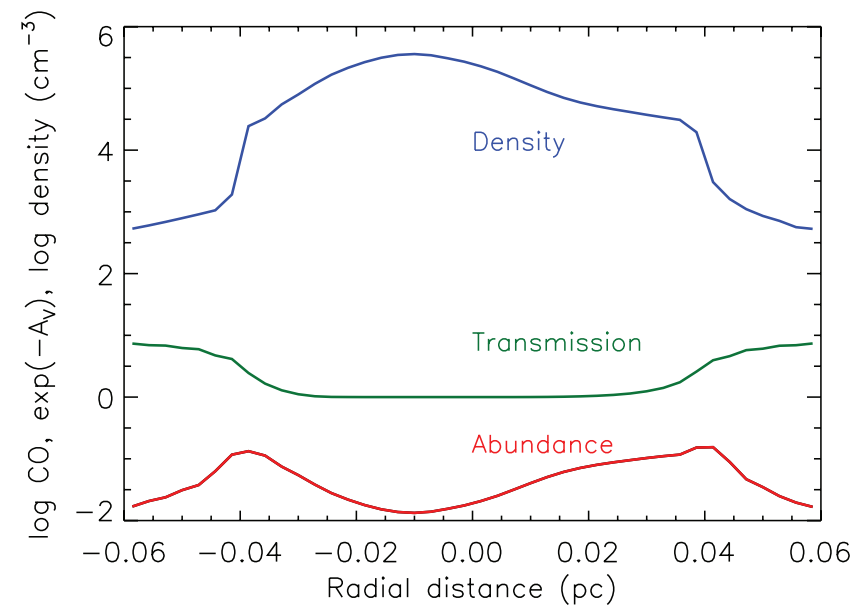

FIG. 4.-Structure of a thermally subcritical core. The figure shows the CO abundance, mean extinction, and gas density in the same format as Fig. 3. Here the model has the parameters of a stable, starless, thermally subcritical core. The asymmetries are perturbations from an internal oscillation. The oscillations are further illustrated in Figs. 6 and 7 of Broderick et al. (2007). Parameters are listed in Table 1.

effects, and improve this model by including variations in the cooling rate due to abundance variations and by including photoelectric heating at the cloud boundary.

\subsection{Line Cooling with Abundance Variations}

In a model for molecular line cooling that uses cooling coefficients based on a local approximation, such as the large velocity gradient (LVG) radiative transfer model (Goldsmith 2001), it is straightforward to include the effect of abundance variations due to depletion. To do this, we use the cooling coefficients for different "depletion factors," for example as listed in the tables in Goldsmith (2001). We assume that the abundance of coolants is given by our simple model of the $\mathrm{CO}$ abundance. This follows because most of the molecular line cooling is through transitions of carbon species such as ${ }^{12} \mathrm{CO},{ }^{13} \mathrm{CO}, \mathrm{C}^{18} \mathrm{O}$, and $\mathrm{CS}$ (Goldsmith 2001).

At the cloud edge where the $\mathrm{CO}$ abundance varies due to dissociation we must also include the cooling due to $\mathrm{C}^{+}$, which can be as effective a coolant in photodissociation regions as $\mathrm{CO}$ is in molecular regions. The $\mathrm{C}^{+}$cooling rate is given by (Tielens 2005 , eq. [27])

$$
\begin{aligned}
& \Lambda_{\mathrm{C}+}=3 \times 10^{-27} n\left(\mathrm{C}^{+}\right) n\left(\mathrm{H}_{2}\right)^{2}\left[1+0.42\left(X_{i} / 1 \times 10^{-3}\right)\right] \\
& \times \exp (-92 / T) \operatorname{ergs~} \mathrm{cm}^{-3} \mathrm{~s}^{-1} \text {, }
\end{aligned}
$$

where $X_{i}$ is the ionization fraction, taken to be the abundance relative to $\mathrm{H}$ of $\mathrm{C}^{+}$, or $1.4 \times 10^{-4} \mathrm{C}^{+} / \mathrm{C}_{\text {total }}$.

Figures 5 and 6 show the effect of abundance variations on our two classes of cores. While a decrease in abundance results in a decrease in the cooling rate, the temperatures of the depleted and undepleted cores are about the same. In the thermally supercritical cores, the cooling at the core center is dominated by collisional coupling to the dust and the cooling at the outer radii is dominated by $\mathrm{C}^{+}$. Only at midradii is the cooling primarily through molecular lines. In the thermally subcritical cores, abundance variations also do not change the temperature too much, but for a different reason. The optical depths of the coolants in the thermally subcritical cores are low enough that the gas can always find some transitions with moderate optical depths for effective cooling despite the 


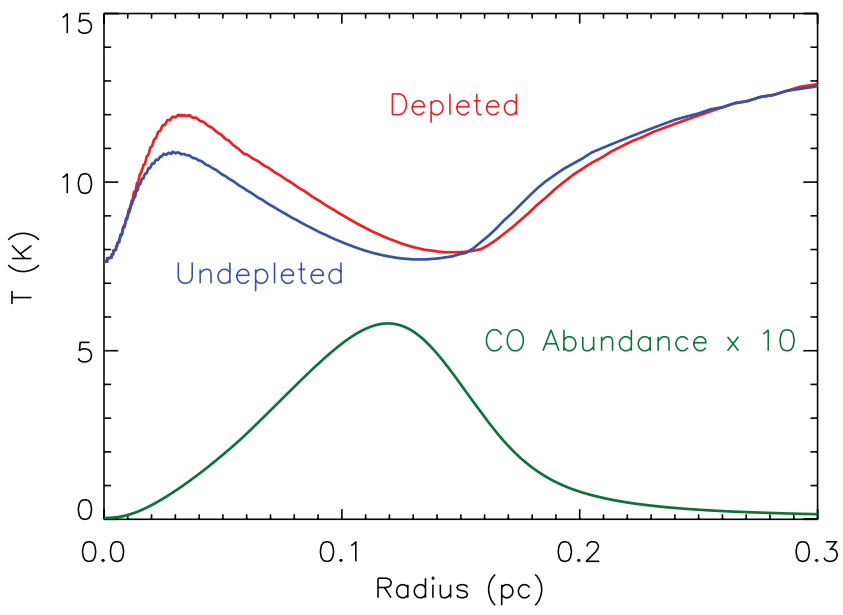

FIG. 5.-Comparison of the temperature of thermally supercritical cores, with and without variable $\mathrm{CO}$ abundances. Although the abundance variations affect the molecular line cooling rate, where the abundance is most reduced the cooling is dominated by the dust or $\mathrm{C}^{+}$rather than the molecular lines. The relative abundance of $\mathrm{CO}$ has been multiplied by 10 so that a value of 10 means undepleted. Parameters are listed in Table 1.

changes in abundance caused by depletion. Pavlyuchenkov et al. (2007) also calculate the effect of depletion on the temperature structure of model starless cores.

\subsection{Photoelectric Heating}

At the edge of the core, high-energy photons can liberate electrons from dust grains by the photoelectric effect. In cold dark clouds, the heating rate is (Bakes \& Tielens 1994; Young et al. 2004)

$$
\Gamma_{\mathrm{pe}}=10^{-24} \epsilon G_{\mathrm{pe}}(r) n\left(\mathrm{H}_{2}\right) \operatorname{ergs~} \mathrm{cm}^{-3} \mathrm{~s}^{-1},
$$

where the efficiency factor $\epsilon=0.5$ for the conditions in cold dark clouds. The factor $G_{\mathrm{pe}}$ is the number of high-energy photons $(h \nu>6 \mathrm{eV})$ normalized by the number in the general interstellar

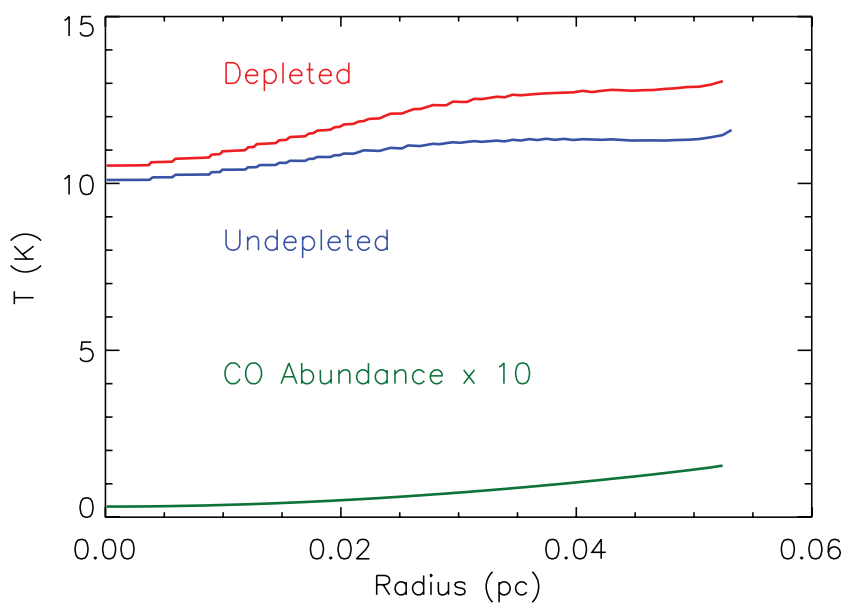

FIG. 6.-Comparison of the temperatures of stable, starless, thermally subcritical cores, with and without variable $\mathrm{CO}$ abundances. These cores are more nearly isothermal than the thermally supercritical cores (Fig. 5) because the gas does not couple with the dust and is cooled primarily by line radiation. The relative abundance of $\mathrm{CO}$ has been multiplied by 10 so that a value of 10 means undepleted. While the abundance of $\mathrm{CO}$ is quite low across the core, there is still a factor of 5 difference between the center and the edge. Parameters are listed in Table 1.

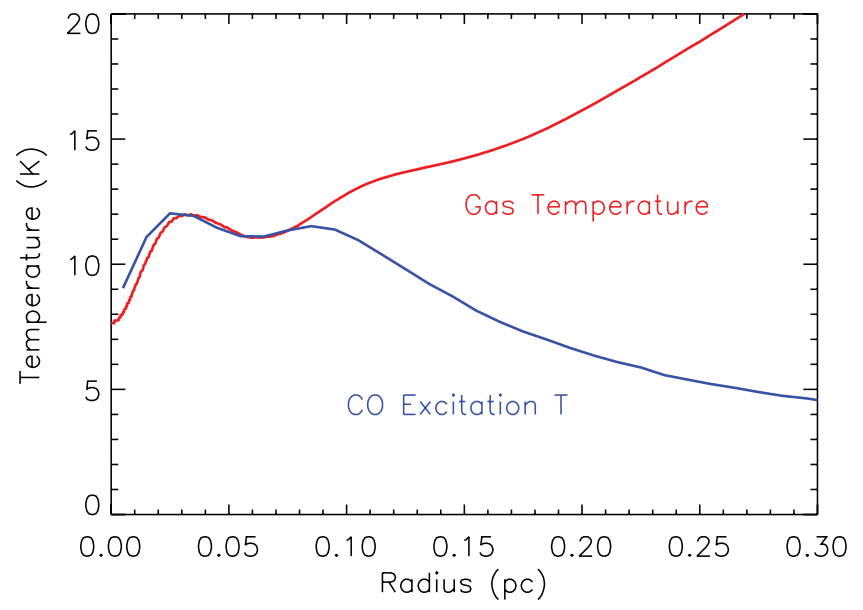

FIG. 7.- Comparison of the gas temperature and the excitation temperature of $\mathrm{CO}(1-0)$ in an unstable, prestellar, thermally supercritical core. Because the gas density (Fig. 3) and abundance of CO (Fig. 5) are so low in the outer core, the excitation temperature of $\mathrm{CO}$ declines with increasing radius even as the gas temperature increases. In the center of the core, the $\mathrm{CO}$ lines are optically thick and the excitation temperature is approximately the same as the gas temperature. Parameters are listed in Table 1.

radiation field. In computing $G_{\mathrm{pe}}(r)$, the intensity is averaged over frequency and direction, $\Omega$,

$$
\begin{aligned}
G_{p e}(r)= & \left(\int_{0}^{4 \pi} \int_{6 \mathrm{eV}}^{\infty} J_{\nu} d \nu d \Omega\right)^{-1} \\
& \times \int_{0}^{4 \pi} \int_{6 \mathrm{eV}}^{\infty} J_{\nu} \exp \left[-\tau_{\nu}(r, \omega)\right] d \nu d \Omega .
\end{aligned}
$$

Here $\tau_{\nu}(r, \omega)$ is the frequency dependent optical depth from $r$ to the cloud surface $r=0$ along some particular direction, $\omega$.

Figures 7 and 8 show the temperature structures of our two classes of cores with photoelectric heating. Where the gas is thin enough that shielding is ineffective, photoelectric heating raises the temperature. Figures 7 and 8 also compare the gas temperature with the excitation temperature of CO computed by our non-LTE radiative transfer code (Keto et al. 2004; Keto \& Field 2005) assuming a total $\mathrm{CO}$ abundance relative to $\mathrm{H}_{2}$ of $5.625 \times 10^{-5}$

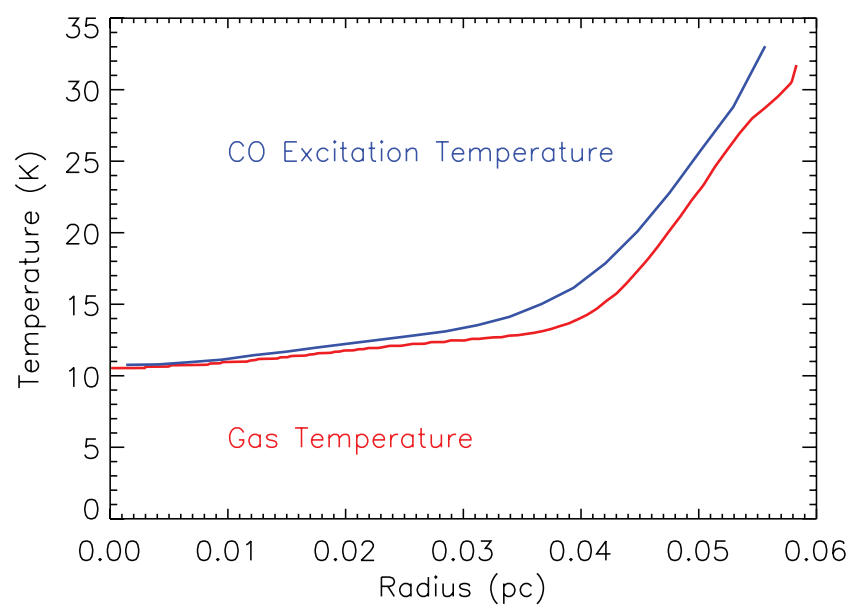

FIG. 8.-Comparison of the gas temperature and the excitation temperature of $\mathrm{CO}$ in a stable, starless, thermally subcritical cores. Because of the low mass of this model $\left(1 M_{\odot}\right)$, the core is truncated at a high enough density that the excitation temperature of the $\mathrm{CO}$ remains close to the gas temperature up to the boundary. Parameters are listed in Table 1. 


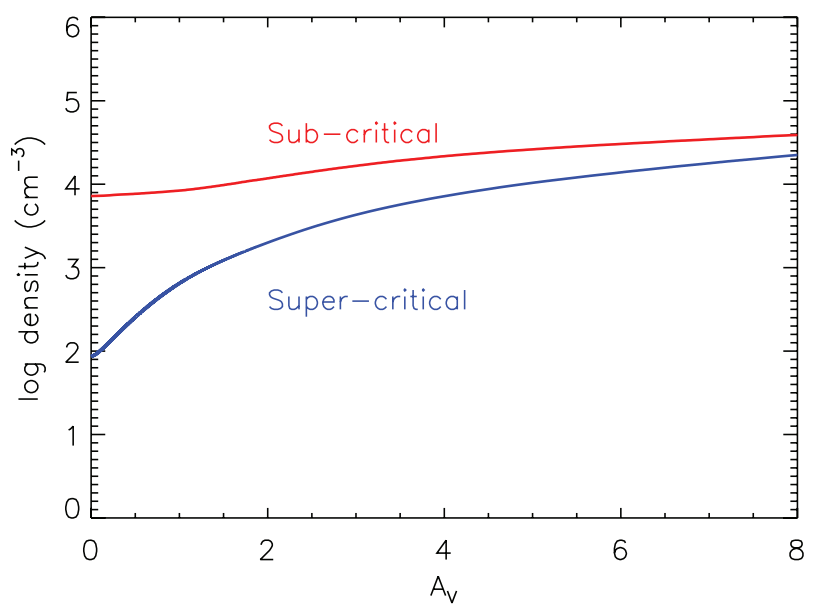

FIG. 9.-Density structure of unstable, prestellar, thermally supercritical cores, compared to the density of stable, starless, thermally subcritical cores. The subcritical cores are truncated at a high density, as would be appropriate for low-mass cores in a high-pressure environment. The density of the thermally supercritical cores decreases to a much lower value at the edge, consistent with a lower surrounding pressure. Parameters are listed in Table 1.

(Goldsmith 2001). In the thermally supercritical cores, the gas density is very low at the boundary (Fig. 9), and the $\mathrm{CO}$ is not strongly excited by collisions. Thus the excitation temperature of $\mathrm{CO}$ declines with radius despite the increase in the gas temperature. This is consistent with observations of the diffuse ISM that show $\mathrm{H}$ spin temperatures of about $75 \mathrm{~K}$ and $\mathrm{CO}$ temperatures below $5 \mathrm{~K}$ (Burgh et al. 2007; Pineda et al. 2008). In contrast, because of the high density throughout the subcritical cores (Fig. 9), the $\mathrm{CO}$ is always collisionally excited and the $\mathrm{CO}$ excitation temperature is everywhere approximately the same as the gas temperature. The $\mathrm{CO}$ excitation temperature slightly exceeds the local gas temperature at locations where the gas temperature is rising rapidly and the $\mathrm{CO}$ line has a non-negligible optical depth. This is because the line radiation from the nearby warmer gas heats the local CO level populations.

Observationally, the excitation temperature of $\mathrm{CO}$ in cores may be determined by comparing the brightness of the (2-1) and (1-0)

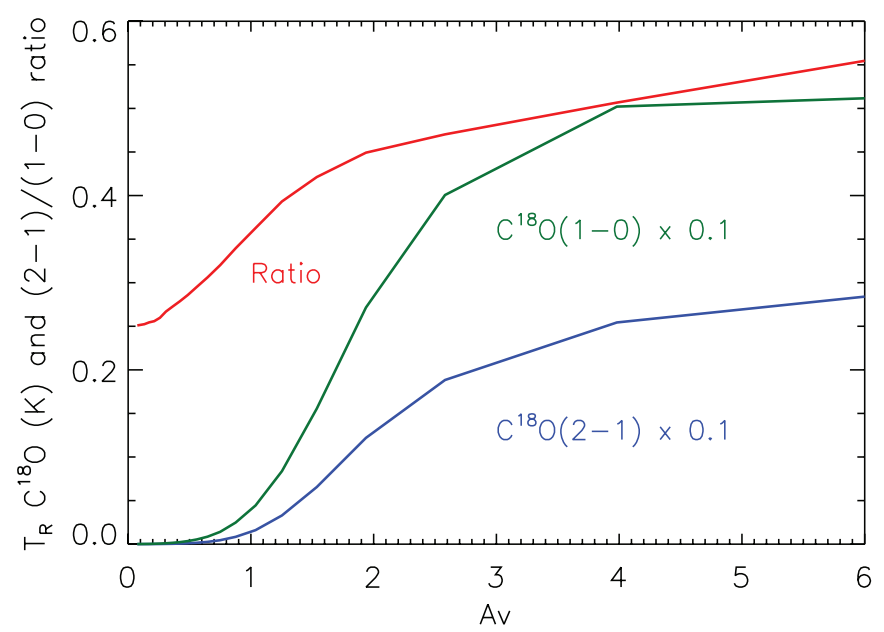

FIG. 10.- Line intensity of $\mathrm{C}^{18} \mathrm{O}(1-0)$ and $\mathrm{C}^{18} \mathrm{O}(2-1)$ (radiation temperature) and their ratio $(2-1) /(1-0)$ in unstable, prestellar thermally supercritical cores. The temperature structure for this model is shown in Fig. 7. The density structure is shown in Fig. 9. The ratio of the $\mathrm{CO}$ lines is a function of the excitation temperature of the $\mathrm{CO}$. In this model because of the low densities at the edge of the core, the $\mathrm{CO}$ excitation temperature is much lower than the gas temperature. Parameters are listed in Table 1.

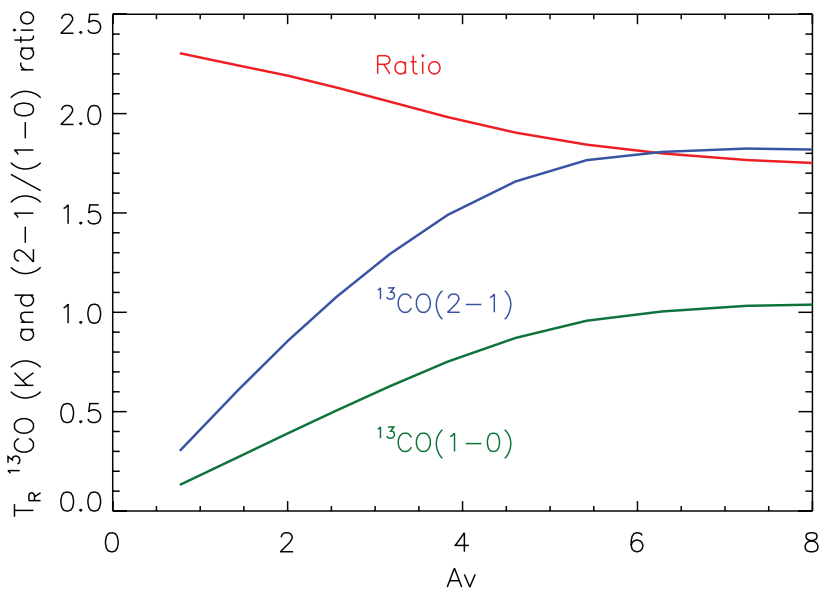

FIG. 11. - Line intensity of ${ }^{13} \mathrm{CO}(1-0)$ and ${ }^{13} \mathrm{CO}(2-1)$ (radiation temperature) and their ratio $(2-1) /(1-0)$ in stable, starless thermally subcritical cores. The temperature structure for this model is shown in Fig. 8. The density structure is shown in Fig. 9. The ratio of the $\mathrm{CO}$ lines is a function of the excitation temperature of the CO. In this model because of the high densities at the edge of the core, the $\mathrm{CO}$ excitation temperature is always close to the gas temperature. Parameters are listed in Table 1.

transitions. Figures 10 and 11 show that the ratio of $\mathrm{CO}(2-1)$ to $\mathrm{CO}(1-0)$ declines at the edge of the thermally supercritical cores as the excitation temperature decreases, whereas in the thermally subcritical cores the ratio rises at the edge. This predicted decrease in the $\mathrm{CO}$ excitation temperature has been observed at the edge of TMC-1C (Fig. 12 of Schnee et al. 2007), a core whose properties put it in the thermally supercritical class. In contrast, at the edge of the subcritical core $\mathrm{B} 68$, the ratio of the $\mathrm{CO}$ lines is observed to be constant or possibly slightly rising (Fig. 5 of Bergin et al. 2006). Although the decreasing line strengths adversely affect the signal-to-noise ratio just at the edge where the comparison is the most diagnostic, the observed ratios are consistent with the different behavior of the line ratios expected in each of the two classes of cores.

\section{IMPLICATIONS}

\subsection{The Rate of Cosmic-Ray Ionization and the Opacity of Fluffy Dust}

Recent observations show promise of measuring the gas temperature with a precision of a few degrees. Such precision might allow a determination of the rate of cosmic-ray ionization and also the opacity of the dust. In dark clouds and dense cores, observations of molecular ions as well as measurements of kinetic temperature limit the possible values of the cosmic-ray ionization rate to between about $1 \times 10^{-17}$ and $6 \times 10^{-17} \mathrm{~s}^{-1}$ (van der Tak \& van Dishoeck 2000; Dalgarno 2006). In our models we compare three different rates, $1.3 \times 10^{-17} \mathrm{~s}^{-1}$ (Spitzer \& Tomasko 1968), $3.0 \times 10^{-17} \mathrm{~s}^{-1}$, and $6.0 \times 10^{-17} \mathrm{~s}^{-1}$, which we refer to as as low, standard, and high cosmic-ray ionization rates. Figures 12 and 13 show the temperature structure for these three different rates in our two classes of cores. In the thermally supercritical cores, the cosmic rays most strongly affect the gas temperature at midradii where the energy input from cosmic rays is significant in the thermal balance. In the core center where the gas temperature is collisionally coupled to the dust, the cosmic rays have little effect because they carry much less energy than the dust radiation. At outer radii the dominant energy source is hot photoelectrically released electrons. The "high" rate produces temperatures at midradii that are higher than generally indicated by observations (Tafalla et al. 2004; Young et al. 2004; Crapsi et al. 2007). 


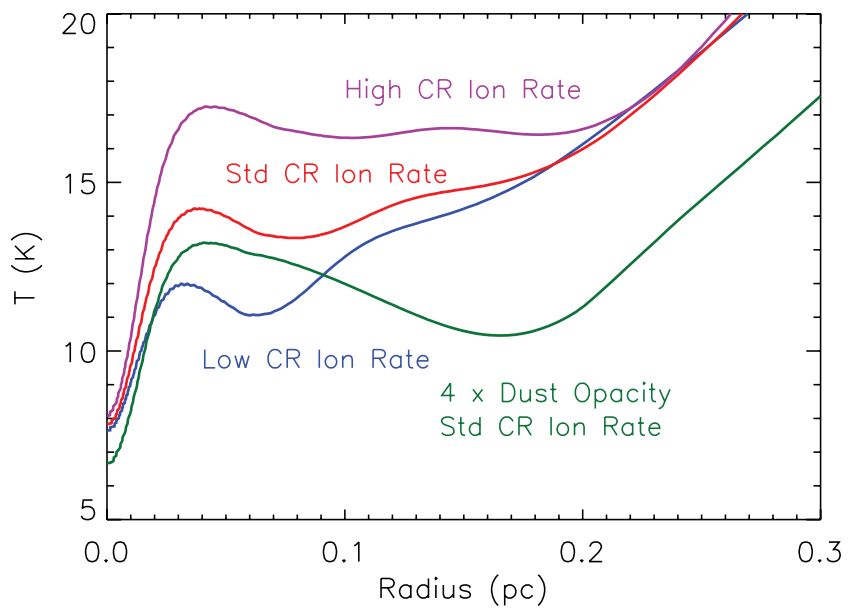

FIG. 12.-Gas temperature in thermally supercritical cores under different conditions. The figure shows the gas temperature for a "low" cosmic-ray ionization rate of $1.3 \times 10^{-17} \mathrm{~s}^{-1}$, a "standard" rate of $3.0 \times 10^{-17} \mathrm{~s}^{-1}$, and a "high" rate of $6.0 \times 10^{-17} \mathrm{~s}^{-1}$. Because cosmic rays heat the gas directly, their rate affects the gas temperature in the intermediate region where the cooling is not dominated by dust and the heating is not dominated by hot photoelectric electrons. The figure also shows the gas temperature if the dust opacity is 4 times the "standard" value. This increase in dust opacity lowers the gas temperature everywhere, in the center and midradii by lowering the dust temperature, and at outer radii by increasing the shielding against high-energy photons that generate hot electrons. Parameters are listed in Table 1.

However, higher sensitivity ammonia observations at core radii of $0.05-0.1 \mathrm{pc}$ are needed to make a definitive statement. In the thermally subcritical cores, because the temperature is nearly uniform except at the edge, and because the gas and dust are not well coupled, the higher cosmic ray rates simply raise the gas temperature across the cores.

The gas temperature is also dependent on the dust opacity. From a comparison of $\mathrm{CO}$ and dust observations, Krüegel \& Siebenmorgen (1994) found that the opacity of dust in dark clouds is higher than in the more diffuse ISM. In the cold quiescent interiors of dark clouds, interstellar dust grains acquire thick icy mantles that increase their opacity. In the high-density centers of thermally supercritical cores, the ice-coated dust may coagulate and become fluffy, further increasing the opacity (see also Ossenkopf \& Henning 1994). Further evidence for higher dust opacities is provided by observations by Evans et al. (2001) that show that a standard dust opacity results in core masses that are beyond the gravitational stability limit if the cores were modeled as BE spheres. If the dust opacity were higher than the standard opacities in the ISM, then the core masses would be less, and the cores more stable with lifetimes longer than free-fall times and more consistent with the numbers of cores observed. Keto et al. (2004) determined the core masses independently of the dust opacity from observations of $\mathrm{N}_{2} \mathrm{H}^{+}$lines and found indications that the dust mass opacity appropriate for the more diffuse ISM is too low for cloud cores.

Precise measurements of the gas temperature in starless cores could provide yet another line of evidence for or against fluffy dust. Figures 12 and 13 compare the gas temperatures in our two classes of cores calculated with the standard dust opacities of Ossenkopf \& Henning (1994) and with the opacities increased by a factor of 4 . The increased opacities reduce the gas temperature everywhere. In the center of the cores, the gas temperature is reduced because the radiative input to the dust is decreased. At the outer radii, the increased dust opacity provides shielding against the high-energy photons responsible for the photoelectric heating.

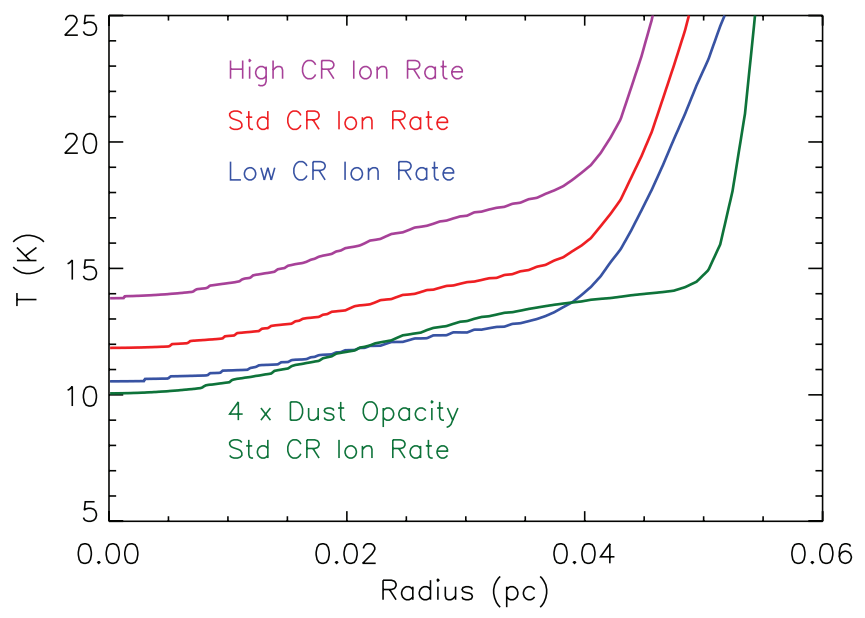

FIG. 13.- Same as Fig. 12 except that the model here is for stable starless thermally subcritical cores, rather than supercritical. Parameters are listed in Table 1.

Although there are complicating factors, the temperatures determined from molecular line observations are generally more consistent with the lower gas temperatures predicted by the increased dust mass opacities of fluffy dust. This is particularly noticeable in the center of the thermally supercritical cores where the observed temperature, about $6 \mathrm{~K}$, (Fig. 4 of Crapsi et al. 2007) is below that obtainable with standard dust opacities even with the "low" rate of cosmic-ray heating.

Another way to raise the dust optical depth without changing the dust opacity is to raise the gas density. If the density of the core were higher, the dust optical depth would also be higher, even for the same mass, and the central temperatures therefore lower. Our model assumes a central density of $1 \times 10^{6} \mathrm{~cm}^{-3}$, a little less than estimated for L1544 by Crapsi et al. (2007). In Figure 12 we present the results for our lower density to maintain consistency throughout our calculations. It is simple enough to compute temperatures for models with higher densities, and the results show that a model with a central density a little over $10^{7} \mathrm{~cm}^{-3}$ is required to bring the central temperature down to $6 \mathrm{~K}$ if we use the standard opacities and cosmic-ray ionization rate. A higher central density of course implies a higher density throughout the core, and this would not be consistent with continuum and molecular line observations of L1544.

Although our models assume a dust opacity that is independent of density, the opacity in starless cores may be higher at densities above $10^{5} \mathrm{~cm}^{-3}$ (Ossenkopf \& Henning 1994). In order to put stringent constraints on the cosmic-ray ionization rate, accurate measurements of the gas temperature as well as the dust continuum emission at various wavelengths will be needed to determine the temperature and dust opacity as a function of radius and density.

\subsection{The Dynamical Stability of Starless Cores}

The starless cores are generally successfully modeled as BE spheres with the addition of some nonthermal energy amounting to a fraction of the total internal energy (Barranco \& Goodman 1998; Goodman et al. 1998; Tafalla et al. 2004; Lada et al. 2008). Any disagreements with observations seem resolvable by the addition of further physical processes to the models. As suggested in our study here, some of these details are significant, such as non-isothermal gas, variable molecular abundances, and perturbations in velocity and density, but so far no observations or theoretical considerations have suggested a revision of the basic model that the cores are pressure-supported, self-gravitating clouds. 


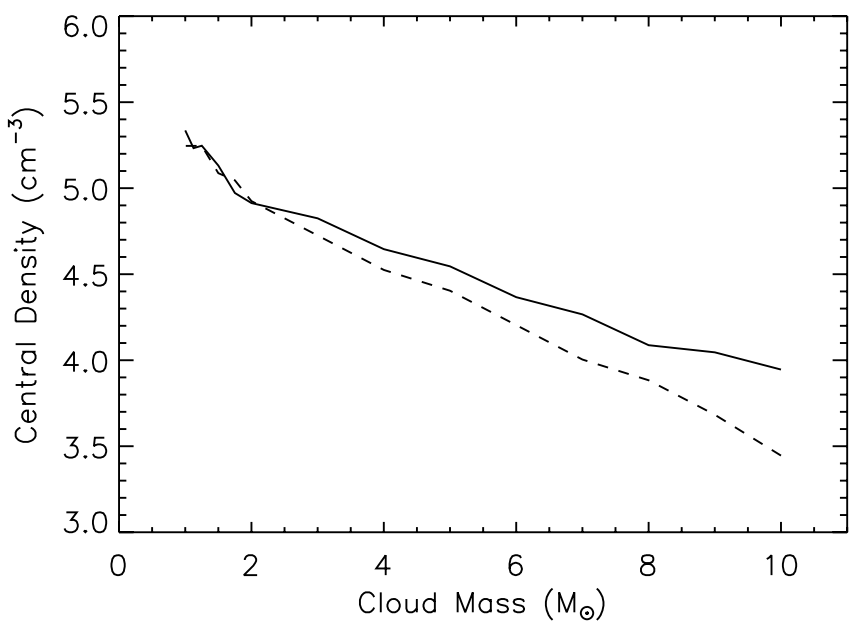

FIG. 14.-Maximum stable central density of a starless core with (solid line) and without (dashed line) photoelectric heating at the core boundary. For the mass indicated on the abscissa, the log of the maximum density in the center of the core is indicated on the ordinate. The figure shows that the inclusion of the photoelectric heating at the core edge marginally improves the stability of the more massive cores. The cores here are modeled with the only internal energy being thermal. The addition of some nonthermal energy in the form of microturbulence or magnetic energy would raise the curves to higher density.

However, the BE spheres as theoretical objects have requirements that are not necessarily met by the starless cores. As truncated solutions of the Lane-Emden equation, the BE spheres require an exterior bounding pressure. If the bounding medium is cold enough that its own self-gravity is significant, then the combination of the BE sphere and the confining medium cannot be dynamically stable. However, if the external medium is hot enough that self-gravitational forces are negligible, then the bounding medium itself will not collapse onto the sphere and decrease its stability. This seems to be the case for isolated cores such as B68. What about cores that exist in the midst of a star-forming region filled with widespread, low-density, molecular gas? Is the increase in temperature of the molecular gas caused by photoelectric heating sufficient to stabilize the surrounding molecular gas?

To investigate this possibility we calculate the maximum stable central densities for cores of different masses with and without photoelectric heating. This calculation assumes the stability criterion of BE spheres (Bonnor 1956) applied to non-isothermal cores. As a function of mass we compute the maximum or critically stable central density, which occurs at the maximum stable external pressure as indicated by a diagram such as Figure 1 of Keto \& Field (2005) or Figure 1 of Lombardi \& Bertin (2001). Figure 14 shows that photoelectric heating improves the stability of larger cores. However, according to Figure 14 the maximum stable density is still lower than the central densities suggested by observations. This does not mean that observed cores are necessarily unstable. The addition of nonthermal energies such as oscillations, turbulence, or magnetic fields that are not included in this calculation might resolve the difference (Galli et al. 2002; Keto \& Field 2005; Keto et al. 2006; Broderick et al. 2007; Hennebelle \& Fromang 2007). However, to include this energy in the model we would need to know how it affects the equation of state. If the equation of state remains as $P \sim \rho^{1}$, then the effect on the stability of the additional nonthermal internal energy is the same as increasing the gas temperature (Keto \& Field 2005). The calculations for Figure 14 suggest that, consistent with observations, the additional energy need only be a fraction of the thermal energy. The analysis suggests that the increased temperature due to photoelectric heating is significant in improving the stability of the more massive cores, but not significant enough to eliminate the need for some additional internal energy.

\section{CONCLUSIONS}

The structure of starless cores has been revisited, taking into account a simple $\mathrm{CO}$ chemistry and photoelectric heating in the radiative energy balance. The main conclusions of this work are as follows.

1. Observations of the different properties of different starless cores can be understood in the context of a simple physical model that shows different structures and behavior depending on whether the central density is above or below about $10^{5} \mathrm{~cm}^{-3}$. This value depends on other properties, in particular masses in the range of $1-10 M_{\odot}$ and temperatures around $10 \mathrm{~K}$, typical values for starless cores.

2 . The low gas temperature $(\leq 7 \mathrm{~K})$ recently observed toward the center of prestellar cores can only be reproduced if the dust opacity is increased by a factor of a few, suggesting that dust grains become fluffy in cold dark cores where the densities are $\geq 10^{5} \mathrm{~cm}^{-3}$.

3. The gas temperature of starless cores is quite sensitive to small changes in the cosmic-ray ionization rate, $\zeta$, at volume densities below about $10^{5} \mathrm{~cm}^{-3}$ (the critical density for gas-dust coupling) and at extinction values larger than about 1 mag (where photoelectric heating is negligible). Thus, high-sensitivity ammonia observations of starless cores can put stringent constraints on $\zeta$. Recent observations toward L1544 suggest $\zeta \simeq 1 \times 10^{-17} \mathrm{~s}^{-1}$.

4. Photoelectric heating affects the stability of a core so that it can support a higher central density or a higher external pressure. However, the effect is only important for the larger starless cores, which still require some additional internal energy to be dynamically stable.

\section{REFERENCES}

Aguti, E. D., Lada, C. J., Bergin, E. A., Alves, J. F., \& Birkinshaw, M. 2007, ApJ, 665, 457

Aikawa, Y., Herbst, E., Roberts, H., \& Caselli, P. 2005, ApJ, 620, 330

Aikawa, Y., Ohashi, N., \& Herbst, E. 2003, ApJ, 593, 906

Aikawa, Y., Ohashi, N., Inutsuka, S., Herbst, E., \& Takakuwa, S. 2001, ApJ, 552,639

Alves, J., Lada, C., \& Lada, E. 2001, Nature, 409, 159

André, P., Basu, S., \& Inutsuka, S.-I. 2008, preprint (arXiv:0801.4210)

Bacmann, A., André, A. P., Puget, J.-L., Abergel, A., Bontemps, S., \& WardThompson, D. 2000, A\&A, 361, 555

Bacmann, A., Lefloch, B., Ceccarelli, C., Steinacker, J., Castets, A., \& Loinard, L. 2002, A\&A, 389, L6

2003, ApJ, 585, L55

Bakes, E. L. O., \& Tielens, A. G. G. M. 1994, ApJ, 427, 822

Barranco, J. A., \& Goodman, A. A. 1998, ApJ, 504, 207
Beichman, C., Myers, P., Emerson, J., Harris, S., Mathieu, R., Benson, P., \& Jennings, R. 1986, ApJ, 307, 337

Benson, P., \& Myers, P. 1989, ApJS, 71, 89

Bergin, E., Alves, J., Huard, T., \& Lada, C. 2002, ApJ, 570, L101

Bergin, E., Ciardi, D., Lada, C., Alves, J., \& Lada, E. 2001, ApJ, 557, 209

Bergin, E., Langer, W., \& Goldsmith, P. 1995, ApJ, 441, 222

Bergin, E. A., Maret, S., van der Tak, F. F. S., Alves, J., Carmody, S. M., \& Lada, C. J. 2006, ApJ, 645, 369

Bergin, E. A., \& Tafalla, M. 2007, ARA\&A, 45, 339

Bonnor, W. 1956, MNRAS, 116, 351

Broderick, A. E., Keto, E., Lada, C. J., \& Narayan, R. 2007, ApJ, 671, 1832

Brown, P., Charnley, S., \& Millar, T. 1988, MNRAS, 231, 409

Burgh, E. B., France, K., \& McCandliss, S. R. 2007, ApJ, 658, 446

Carolan, P. B., Redman, M. P., Keto, E., \& Rawlins, J. M. C. 2008, MNRAS, 383,705 
Caselli, P., Walmsley, C., Tafalla, M., Dore, L., \& Myers, P. 1999, ApJ, 523, L165 Caselli, P., Walmsley, C., Zucconi, A., Tafalla, M., Dore, L., \& Myers, P. 2002, ApJ, 565, 344

Clarke, C. J., \& Pringle, J. E. 1997, MNRAS, 288, 674

Collins, M. P., Dever, J. W., Fraser, H. J., \& McCoustra, M. R. S. 2003a, Ap\&SS, 285, 633

Collins, M. P., Dever, J. W., Fraser, H. J., McCoustra, M. R. S., \& Williams, D. A. 2003b, ApJ, 583, 1058

Crapsi, A., Caselli, P., Walmsley, C. M., Myers, P. C., Tafalla, M., Lee, C. W., \& Bourke, T. L. 2005, ApJ, 619, 379

Crapsi, A., Caselli, P., Walmsley, C. M., \& Tafalla, M. 2007, A\&A, 470, 221

Crapsi, A., Caselli, P., Walmsley, C. M., Tafalla, M., Lee, C. W., Bourke, T. L., \& Myers, P. C. 2004, A\&A, 420, 957

Dalgarno, A. 2006, Proc. Natl. Acad. Sci., 103, 12269

di Francesco, J., Evans, N. J., II, Caselli, P., Myers, P. C., Shirley, Y., Aikawa, Y., \& Tafalla, M. 2007, in Protostars and Planets V, ed. B. Reipurth, D. Jewitt, \& K. Keil (Tucson: Univ. Arizona Press), 17

Evans, N., Rawlings, J., Shirley, Y., \& Mundy, L. 2001, ApJ, 557, 193

Fraser, H. J., Collings, M. P., McCoustra, M. R. S., \& Williams, D. A. 2001, MNRAS, 327, 1165

Galli, D., Walmsley, M., \& Gonçalves, J. 2002, A\&A, 394, 275

Goldsmith, P. 2001, ApJ, 557, 736

Goncalves, J., Galli, D., \& Walmsley, M. 2004, A\&A, 415, 617

Goodman, A. A., Barranco, J. A., Wilner, D. J., \& Heyer, M. H. 1998, ApJ, 504,223

Gregersen, E., \& Evans, N. 2000, ApJ, 538, 260

Gregersen, E., Evans, N., Zhou, S., \& Choi, M. 1997, ApJ, 484, 256

Habing, H. J. 1968, Bull. Astron. Inst. Netherlands, 19, 421

Hasegawa, T., \& Herbst, E. 1993, MNRAS, 261, 83

Hasegawa, T., Herbst, E., \& Leung, C. 1992, ApJS, 82, 167

Hennebelle, P., \& Fromang, S. 2007, preprint (arXiv:0709.2886)

Hotzel, S., Harju, J., \& Juvela, M. 2002, A\&A, 395, L5

Keto, E., Broderick, A., Lada, C. J., \& Narayan, R. 2006, ApJ, 652, 1366

Keto, E., \& Field, G. 2005, ApJ, 635, 1151

Keto, E., Rybicki, G., Bergin, E., \& Plume, R. 2004, ApJ, 613, 355

Kirk, J. M., Ward-Thompson, D., \& André, P. 2005, MNRAS, 360, 1506

Krüegel, E., \& Siebenmorgen, R. 1994, A\&A, 288, 929

Lada, C. J., Bergin, E. A., Alves, J. F., \& Huard, T. L. 2003, ApJ, 586, 286

Lada, C. J., Muench, A. A., Rathborne, J., Alves, J. F., \& Lombardi, M. 2008, ApJ, 672, 410

Larson, R. 1973, Fundam. Cosmic Phys., 1, 1 1985, MNRAS, 214, 379

Launhardt, R., Evans, N., Wang, Y., Clemens, D., Henning, T., \& Yun, J. 1998, ApJS, 119, 59

Lee, C., Myers, P., \& Tafalla, M. 1999, ApJ, 526, 788 2001, ApJS, 136, 703

Lee, C. W., \& Myers, P. C. 1999, ApJS, 123, 233

Lee, C. W., Myers, P., \& Plume, R. 2004a, J. Korean Astron. Soc., 37, 257 2004b, ApJS, 153, 523

Lee, J., Bergin, E., \& Evans, N. 2004, ApJ, 617, 360

Li, Z.-Y., Shematovich, V. I., Wiebe, D. S., \& Shustov, B. M. 2002, ApJ, 569, 792

Lombardi, M., \& Bertin, G. 2001, A\&A, 375, 1091

Maret, S., Bergin, E. A., \& Lada, C. J. 2007, ApJ, 670, L25

Mathis, J. S., Rumpl, W., \& Nordsieck, K. H. 1977, ApJ, 217, 425

Myers, P., \& Benson, P. 1983, ApJ, 266, 309

Myers, P., Linke, R., \& Benson, P. 1983, ApJ, 264, 517

Öberg, K. T., Fuchs, G. W., Awad, Z., Fraser, H. J., Schlammer, S., van Dishoeck, E. F., \& Linnatz, H. 2007, ApJ, 662, L23
Öberg, K. T., van Broekhuizan, F., Fraser, H. J., Bisschop, S. E., \& van Dishoeck, E. F. 2005, ApJ, 621, L33

Ossenkopf, V., \& Henning, T. 1994, A\&A, 291, 943

Pagani, L., Bacmann, A., Cabrit, S., \& Vastel, C. 2007, A\&A, 467, 179

Pagani, L., Pardo, J.-R., Apponi, A. J., Bacmann, A., \& Cabrit, S. 2005, A\&A, 429,181

Pagani, L., et al. 2003, A\&A, 406, L59 2004, A\&A, 417, 605

Pavlyuchenkov, Ya., Henning, Th., \& Wiebe, D. 2007, ApJ, 669, L101

Pavlyuchenkov, Y. N., Shustov, B. M., Shematovich, V. I., Wiebe, D. S., \& Li, Z.-Y. 2003, Astron. Rep., 47, 176

Pineda, J. E., Caselli, P., \& Goodman, A. A. 2008, ApJ, 679, 481

Rawlins, J. M. C., Hartquist, T. W., Menten, C. M., \& Williams, D. A. 1992, MNRAS, 255, 471

Redman, M. P., Keto, E., \& Rawlings, J. M. C. 2006, MNRAS, 370, L1

Redman, M. P., Rawlings, J. M. C., Nutter, D. J., Ward-Thompson, W., \& Williams, D. A. 2002, MNRAS, 337, L17

Roberts, J. F., Rawlings, J. M. C., Viti, S., \& Williams, D. A. 2007, MNRAS, 382,733

Schnee, S., Caselli, P., Goodman, A., Arce, H. G., Ballesteros-Paredes, J., \& Kuchibhotla, K. 2007, ApJ, 671, 1839

Schnee, S., \& Goodman, A. 2005, ApJ, 624, 254

Shematovich, V. I., Wiebe, D. S., Shustov, B. M., \& Li, Z.-Y. 2003, ApJ, 588, 894

Shirley, Y., Evans, N., \& Rawlings, J. 2002, ApJ, 575, 337

Shirley, Y., Evans, N., Rawlings, J., \& Gregersen, E. 2000, ApJS, 131, 249

Sohn, J., Lee, C. W., Lee, H. M., Park, Y.-S., Myers, P. C., Lee, Y., \& Tafalla, M. 2004, J. Korean Astron. Soc., 37, 261

Sohn, J., Lee, C. W., Park, Y.-S., Lee, H. M., Myers, P. C., \& Lee, Y. 2007, ApJ, 664, 928

Spitzer, L. J., \& Tomasko, M. G. 1968, ApJ, 152, 971

Stamatellos, D., \& Whitworth, A. 2003, A\&A, 407, 941

Steinacker, J., Bacmann, A., Henning, Th., \& Klessen, R. 2005a, in Protostars and Planets V, ed. B. Reipurth, D. Jewitt, \& K. Keil (Tucson: Univ. Arizona Press), 8107

Steinacker, J., Bacmann, A., Henning, Th., Klessen, R., \& Stickel, M. 2005b, A\&A, 434, 167

Tafalla, M., Mardones, D., Myers, P., Caselli, P., Bachiller, R., \& Benson, P. 1998, ApJ, 504, 900

Tafalla, M., Myers, P., Caselli, P., \& Walmsley, M. 2004, A\&A, 416, 191

Tafalla, M., Myers, P., Caselli, P., Walmsley, C., \& Comito, C. 2002, ApJ, 569, 815

Tafalla, M., Santiago-García, J., Myers, P. C., Caselli, P., Walmsley, C. M., \& Crapsi, A. 2006, A\&A, 455, 577

Tielens, A. G. G. M. 2005, The Physics and Chemistry of the Interstellar Medium (Cambridge: Cambridge Univ. Press)

Tielens, A. G. G. M., \& Hollenbach, D. 1985, ApJ, 291, 722

van der Tak, F. F. S., \& van Dishoeck, E. F. 2000, A\&A, 358, L79

Ward-Thompson, D., André, P., \& Kirk, J. 2002, MNRAS, 329, 257

Ward-Thompson, D., Motte, F., \& André, P. 1999, MNRAS, 305, 143

Ward-Thompson, D., Scott, P., Hills, R., \& André, P. 1994, MNRAS, 268, 276

Whittet, D. C. B., Shenoy, S. S., Bergin, E. A., Chiar, J. E., Gerakines, P. A., Gibb, E. L., Melnick, G. J., \& Neufeld, D. A. 2007, ApJ, 655, 332

Willacy, K., Langer, W. D., \& Velusamy, T. 1998, ApJ, 507, L171

Willacy, K., \& Williams, D. 1993, MNRAS, 260, 635

Williams, J. P., Myers, P. C., Wilner, D. J., \& DiFrancesco, J. 1999, ApJ, 513, L61

Young, K. E., Lee, J.-E., Evans, N. J., II, Goldsmith, P. F., \& Doty, S. D. 2004 ApJ, 614, 252

Zucconi, A., Walmsley, C., \& Galli, D. 2001, A\&A, 376, 650 